\title{
High-resolution gravity and geoid models in Tahiti obtained from new airborne and land gravity observations: data fusion by spectral combination
}

\author{
Hsuan-Chang Shih ${ }^{1}$, Cheinway Hwang ${ }^{2 *}$, Jean-Pierre Barriot ${ }^{3}$, Maxime Mouyen ${ }^{4}$, Pascal Corréia ${ }^{5}$,
} Didier Lequeux ${ }^{5}$ and Lydie Sichoix ${ }^{3}$

\begin{abstract}
For the first time, we carry out an airborne gravity survey and we collect new land gravity data over the islands of Tahiti and Moorea in French Polynesia located in the South Pacific Ocean. The new land gravity data are registered with GPS-derived coordinates, network-adjusted and outlier-edited, resulting in a mean standard error of $17 \mu \mathrm{Gal}$. A crossover analysis of the airborne gravity data indicates a mean gravity accuracy of $1.7 \mathrm{mGal}$. New marine gravity around the two islands is derived from Geosat/GM, ERS-1/GM, Jason-1/GM, and Cryosat-2 altimeter data. A new 1-s digital topography model is constructed and is used to compute the topographic gravitational effects. To use EGM08 over Tahiti and Moorea, the optimal degree of spherical harmonic expansion is 1500. The fusion of the gravity datasets is made by the band-limited least-squares collocation, which best integrates datasets of different accuracies and spatial resolutions. The new high-resolution gravity and geoid grids are constructed on a 9-s grid. Assessments of the grids by measurements of ground gravity and geometric geoidal height result in RMS differences of $0.9 \mathrm{mGal}$ and $0.4 \mathrm{~cm}$, respectively. The geoid model allows 1-cm orthometric height determination by GPS and Lidar and yields a consistent height datum for Tahiti and Moorea. The new Bouguer anomalies show gravity highs and lows in the centers and land-sea zones of the two islands, allowing further studies of the density structure and volcanism in the region.
\end{abstract}

Keywords: French Polynesia; Geoid; Gravity anomaly; Moorea; Least-squares collocation; Tahiti

\section{Background}

The islands of Tahiti and Moorea were formed by volcanism in response to motion of the Pacific plate over a fixed hot-spot fed by mantle upwelling (Devey et al. 2003; McNutt 1998). They are among the 118 islands and atolls in French Polynesia over an area of active volcanism between $5^{\circ}-30^{\circ} \mathrm{S}$ and $130^{\circ}-160^{\circ} \mathrm{W}$, populated with 268,270 people. Because the island of Tahiti is the economic and political center of French Polynesia, it is important to understand the geological structure and to build a sophisticated geodetic infrastructure in Tahiti and its adjacent islands. The geological understanding and infrastructure building will enable identification of

\footnotetext{
* Correspondence: cheinway@mail.nctu.edu.tw

${ }^{2}$ Department of Civil Engineering, National Chiao Tung University, Hsinchu 300, Taiwan

Full list of author information is available at the end of the article
}

potential spots of natural hazard and allow convenient applications of modern geodetic techniques such as global positioning system (GPS) and light detection and ranging (Lidar) to map such spots. Below, we will use "Tahiti" as the single title when a subject is involved with both the islands of Tahiti and Moorea.

Earlier geophysical studies in Tahiti have been focused on the geological structures of the islands. For example, Leroy (1994) investigated the volcanic structure of Tahiti using gravity data collected at 40 sites in Tahiti (Fig. 1a). Clouard et al. (2000) constructed free-air anomaly and residual isostatic anomaly maps to assess the isostatic states associated with ancient magma chambers in some of the islands in French Polynesia, including Tahiti. Patriat et al. (2002) used Tahiti gravity data as an aid when interpreting the deep crustal structure of Tahiti from seismic data. While the land gravity data from Leroy (1994) are 

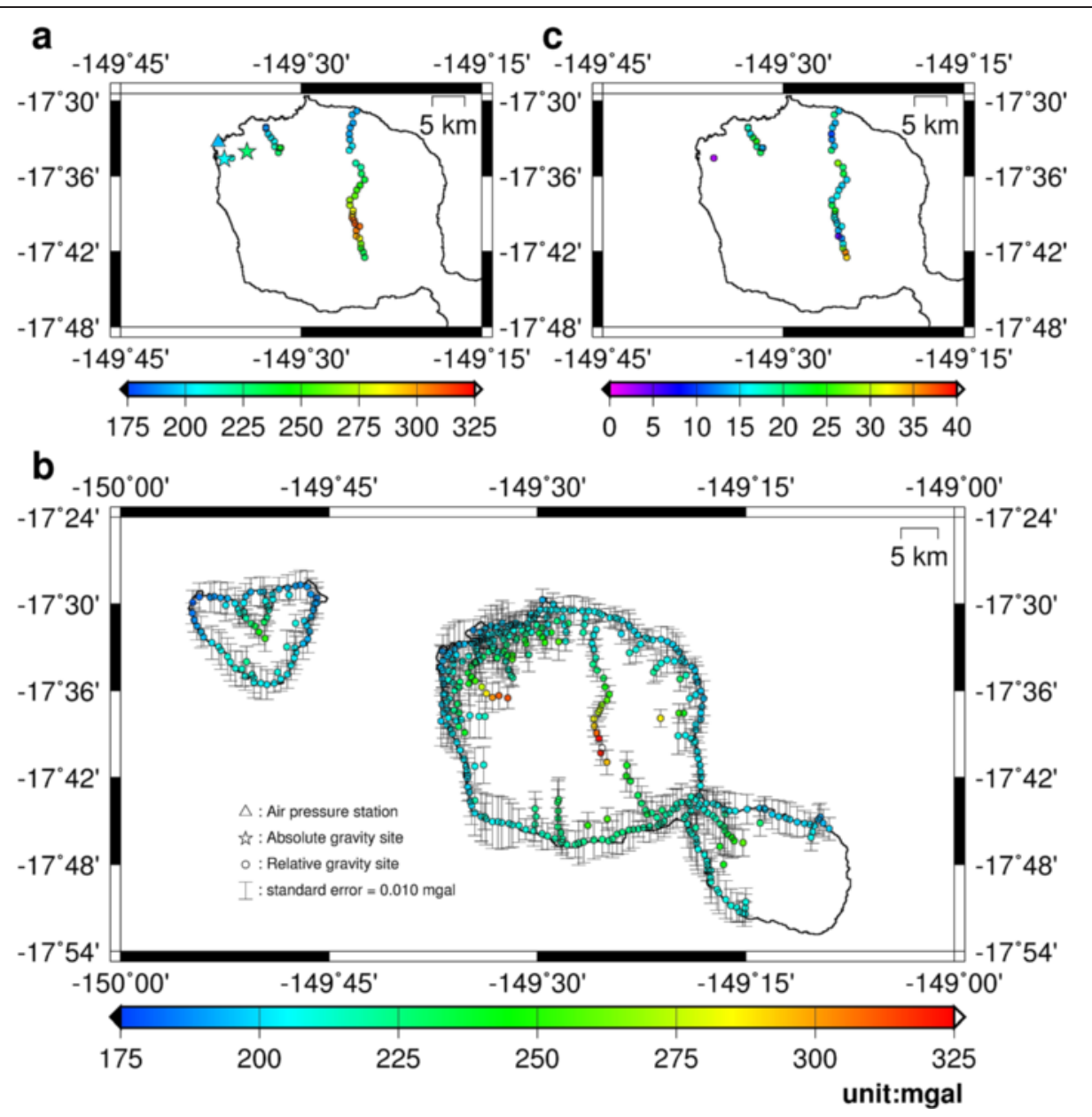

Fig. 1 Land free-air gravity anomalies in Tahiti. a At 40 points, with data collected in 1994. b At 461 points, with data collected in 2013. c Gravity differences (gravity values in 2013 relative to those in 1994). In Fig. 1a, stars represent the two absolute gravity sites and triangle represents the air pressure station, with data collected in 2013. Circles represent relative gravity sites. The vertical bars centered at the circles denote the standard errors of the adjusted gravity values

valuable, the measurements are mainly distributed along a profile from the coast to the center of Tahiti (Fig. 1a), making a limited insight on the three-dimensional structure of the Tahiti volcanism. The insight can be improved by the new gravity datasets collected in this study. In the oceans around Tahiti, gravity-based geophysical investigations have been largely based on marine gravity fields from old-generation altimeter missions and shipborne measurements (Calmant and Cazenave 1987; Sichoix and Bonneville 1996). These studies may be significantly improved by using the high-resolution and high-precision gravity from the new-generation altimeters Jason-1/GM and Cryosat-2 (Sandwell et al. 2014; Hwang and Chang 2014). In addition, there was no specific campaign to collect gravity data for modeling the geoid of Tahiti, which in the past is exclusively derived from global gravity models (GGMs) such as EGM08 (Pavlis et al. 2008).

Because of the importance of a detailed gravity field for Tahiti's mapping infrastructure and Earth science studies, in 2013, the Service de l'Aménagement et de l'Urbanisme of French Polynesia launched campaigns to collect new land and airborne gravity data in Tahiti. The new land and airborne measurements were carried out by the authors in this paper. For the first time, an airborne gravity survey was flown over Tahiti and delivered gravity measurements at a uniform spacing. Although the primary mission of this survey is height modernization of Tahiti through construction of a Tahiti geoid, the product from the mission-airborne gravity values-can also benefit various studies in Tahiti, such as gravity inversion by growing bodies to model subsurface structures (Camacho et al. 2000). In addition, the new land gravity survey occupied sites not only along the major routes across the islands but also the coastal routes around the islands (Fig. 1b). The combination of gravity measurements from these two surveys leads to a completely new gravity dataset in Tahiti.

In this paper, we will accomplish the following two objectives: (1) detailing the data acquisition and processing 
for the 2013 airborne and land gravity surveys, and (2) constructing the detailed gravity anomaly and geoid models of Tahiti by combination of all gravity data available around Tahiti. For the first objective, we show how a small aircraft and an air/sea gravimeter can be used to map the gravity field on islands such as Tahiti and how the resulting gravity field is compared with the existing one. For the second objective, we show how the method of band-limited least-squares collocation (BL-LSC; Moritz 1978; Novák and Heck 2002) is used to combine gravity datasets of different accuracies and spatial resolutions. We also describe how the new gravity anomaly and geoid models of Tahiti are constructed by the remove-computerestore (RCR) procedure, in which the high-frequency components were computed by a new $1 " \times 1$ " digital topography model (DTM, covering both land and sea) of Tahiti. The gravity anomaly and geoid models will be assessed by ground measurements in Tahiti.

\section{Methods}

In this section, we first give an overview of the data used in the present study. We then introduce the methods for the gravity and geoid modeling.

\section{Land gravity data}

The gravity values at the two absolute gravity sites (Fig. 1a) were determined in 2003 using a FG5 absolute gravimeter and the related information is shown in Table 1. With a Scintrex CG5 relative gravimeter, we collected relative gravity values at 461 sites, of which 387 are on the island of Tahiti and 74 on the island of Moorea (Fig. 1b). The land gravity data were corrected for the effects of polar motion, air pressure (data from the Geodesy Observatory of the Tahiti Weather Station), and solid and ocean tides (the latter uses the GOTIC2 software with the NAO99 tide model, Matsumoto et al. 2000; 2001). Afterwards, we carried out a network adjustment of the relative gravity values by holding fixed the gravity values at the two absolute gravity sites (Hwang et al. 2002). The result shows that the mean of the posterior standard errors at the 461 gravity sites is
$17 \mu \mathrm{Gal}$. The posteriori standard error was computed from the prior standard error of a measurement by network adjustment.

Figure 1c shows the differences at the common gravity sites in the 2013 survey (this paper) and the 1994 survey (Leroy 1994). The gravity differences (gravity values in 2013 relative to those in 1994) range from 3 to $35 \mathrm{mGal}$, with a mean value of $17.6 \mathrm{mGal}$. The 2013 and 1994 gravity values are based on two different gravity datums: the datum implied by the new FG5 absolute gravity (for 2013; see Table 1), and the datum implied by the International Gravity Standardization Network 1971 (IGSN71; Morelli et al. 1974). Ihde (1993) also found a difference of $14 \mathrm{mGal}$ between the absolute gravity value and the gravity value from IGSN71 at gravity site Potsdam A. In addition, the elevations of the gravity sites in the 1994 survey were determined by barometric leveling, so that the uncertainties of such elevations will probably increase with elevations. The differences between the gravity values in the 2013 and 1994 surveys were most likely caused by both gravity datum difference and elevation error. Despite the differences, the patterns of gravity variations along the two major profiles are similar (Fig. 1a, b).

\section{Marine gravity from satellite altimeters}

To achieve a best marine gravity field around Tahiti from satellite altimeters, we first improved the altimeter range measurements from the geodetic missions of Geosat, ERS-1, and Jason-1 by re-determining the gates of radar returns using the subwaveform retracker (Yang et al. 2011). The altimeter data from Cryosat- 2 were used without retracking. In general, the accuracy gain in coastal regions due to retracking is larger than the gain in the open oceans. The sea surface heights (SSHs) were then converted to along-track geoid gradients, which were used to form geoid gradients in the north and east directions on a $1^{\prime} \times 1^{\prime}$ grid. Finally, we used the inverse Vening Meinesz formula (Hwang 1998) to compute marine gravity anomalies from the two gradient components. The resulting marine gravity anomaly field (the area defined in

Table 1 Information about the 2003 absolute gravity measurements in Tahiti

\begin{tabular}{|c|c|c|c|c|c|c|}
\hline Gravity site & Gravity value ( $\mu \mathrm{Gal})$ & Coordinates & $\begin{array}{l}\text { Gravity gradient } \\
(\mu \mathrm{Gal} / \mathrm{cm})\end{array}$ & Date & Number of sets ${ }^{a}$ & $\begin{array}{l}\text { Set scatter } \\
(\mu \mathrm{Gal})\end{array}$ \\
\hline \multirow[t]{3}{*}{ Tahiti University } & $978682307.79 \pm 0.64^{b}$ & $17.57680^{\circ} \mathrm{S}$ & -3.00 & 27 June 2003 & 40 & 4.06 \\
\hline & & $149.60620^{\circ} \mathrm{E}$ & & & & \\
\hline & & $94.48 \mathrm{~m}$ & & & & \\
\hline \multirow[t]{3}{*}{ Tahiti LDG } & $978629353.48 \pm 0.52$ & $17.53330^{\circ} \mathrm{S}$ & -3.00 & 30 June 2003 & 65 & 4.19 \\
\hline & & $149.56660^{\circ} \mathrm{E}$ & & & & \\
\hline & & $350.00 \mathrm{~m}$ & & & & \\
\hline
\end{tabular}

\footnotetext{
${ }^{\mathrm{a}}$ One set lasts 30 minutes and contains 100 drops
}

${ }^{\mathrm{b}}$ Measurement precision 
Fig. 2) was assessed by the shipborne gravity measurements from the National Geophysical Data Center (NGDC; http://www.ngdc.noaa.gov/), resulting in a standard deviation of $6.3 \mathrm{mGal}$ in the differences. This level of gravity accuracy is the same as that of the latest gravity field by Sandwell et al. (2014). In Fig. 2, the land gravity anomalies were computed on a $1^{\prime} \times 1^{\prime}$ grid from EGM08 to degree 2160 without contributions from the new airborne and land gravity datasets.

\section{Airborne gravity data}

The Tahiti airborne gravity survey was carried out from July to August, 2013. The field work lasted for about 2 weeks, with a total flight hours of $25 \mathrm{~h}$. The mobile gravimeter collecting the airborne gravity is the LaCoste \& Romberg Air-Sea gravity System II (S-133), supplied by the Ministry of the Interior (MOI) of Taiwan. A Britten-Norman BN-2 Islander aircraft housed the gravimeter and was equipped with a Trimble 5700 GPS receiver. Both the gravimeter and GPS receiver collected readings at the rate of $1 \mathrm{~Hz}$. At speeds of $50-60 \mathrm{~m} / \mathrm{s}$, the aircraft planned to fly at an altitude of $10,000 \mathrm{ft}$ (3048 m). The resulting mean altitude is $3223 \mathrm{~m}$. Table 2 summarizes the key information of the flight lines. With the GPS receiver at the base station of TAHI at the Geodesy Observatory of Tahiti (University of French Polynesia) and the onboard GPS receiver, we used the method of kinematic positioning to determine coordinates of the aircraft. The positioning was accomplished by the software of Trimble Total Control version 2.7, with the GPS orbits from the rapid ephemeris of the International GNSS Service. Because of an open view for the onboard GPS antenna, most of the aircraft coordinates
Table 2 Numbers of flight lines and line spacings of the Tahiti airborne gravity survey

\begin{tabular}{lll}
\hline Direction of flight & Number of flight lines & Line spacing \\
\hline North-south & 17 & $0.05^{\circ}$ \\
East-west & 4 & $0.1^{\circ}$ \\
Northwest-southeast & 4 & $0.1^{\circ}$ \\
\hline
\end{tabular}

were determined to the dm-level accuracy. During the sharp turning of the aircraft to start a new flight line, the gravimeter did not collect data and typically GPS signals were lost due to the high aircraft dynamics. Velocities and accelerations of the aircraft and reductions of the airborne gravity observations were made by the NCTU airborne gravimetry software, see more details in Hwang et al. (2006, 2007). The window size for filtering is $200 \mathrm{~s}$, resulting in a spatial resolution of $5.5 \mathrm{~km}$ at the halfwavelength. Also, the resulting airborne gravity anomalies were derived from a relative gravity point near the parking space of the aircraft.

Figure 3a shows the free-air gravity anomalies at the flight altitude obtained from the Tahiti airborne gravity survey. Due to strong turbulences during the flight, there are no reliable gravity data from flight line No. 4 . The free-air gravity anomalies are highly correlated with the terrain and ocean bottom topography. The maximum value of about $280 \mathrm{mGal}$ is found over the center of the island of Tahiti, and the minimum value of about $20 \mathrm{mGal}$ is over the oceanic area. As Tahiti is created by volcanism, its bulk of mass is under the seas, with only the upper part of the volcano above the sea level. As such, free-air gravity anomalies are positive around Tahiti.

It is a common practice to assess the quality of airborne gravity data by computing the gravity differences

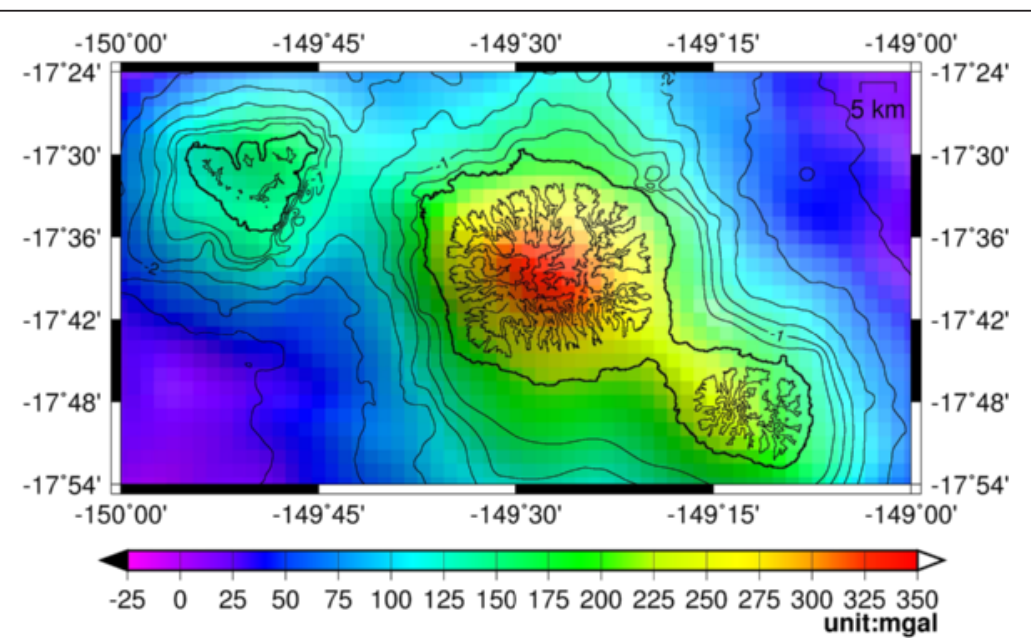

Fig. 2 Free-air gravity anomalies from satellite altimetry over oceans and from EGM08 to degree 2160 on land. The gravity value at a grid point is from EGM08 or altimetry depending on its position on land or ocean. The land (EGM08) values are to be compared with the new gravity anomaly model in Fig. 7. The topographic contours (from the DTM in Fig. 4) are shown at a 500-m interval 


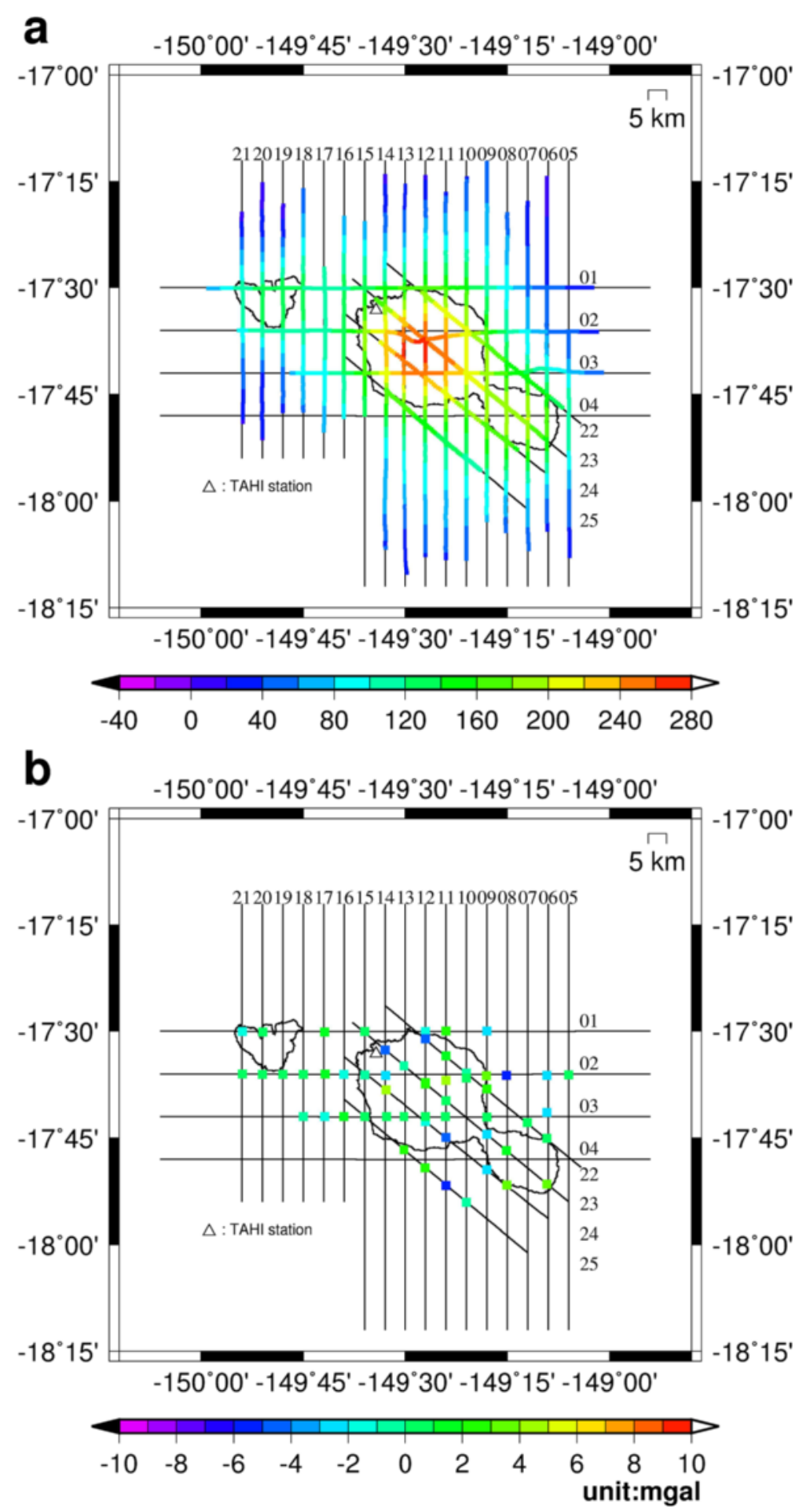

Fig. 3 Results of the Tahiti airborne gravity survey after the crossover adjustment. a Free-air gravity anomalies at the flight altitude. b Gravity differences at crossover points. The numbers near the lines are the flight numbers. The triangle shows the location of the TAHI base station

at the points of any two intersecting lines (crossover points). The differences were determined by subtracting the gravity anomalies of the east-west lines from those of the north-south lines at crossover points. Such gravity differences can also be used to carry out a crossover adjustment for along-line gravity anomaly values (Hwang et al. 2006). We follow this practice to analyze the quality of the Tahiti airborne gravity and to determine a bias 
for each of the survey lines. In this paper, the airborne gravity anomalies along the flight lines No. 19-25 were used as the control points (constraints) in the adjustment, for the smooth flight condition (no turbulence) during the flights. Additionally, there is no need of height correction for the gravity difference at the crossover point because of the insensitivity of gravity anomaly to height difference around the mean flight altitude. Gravity differences larger than $10 \mathrm{mGal}$ were not used in the adjustment; they were usually caused by turbulences during flights. Table 3 shows the statistics of gravity differences at 54 crossover points before and after the crossover adjustment. Figure 3b shows the gravity differences after the adjustment. There is no apparent systematic bias along any of the flight lines. According to Table 3 and Fig. 3b, the averaged standard error of "point" airborne gravity anomalies (unlike land gravity values, the final airborne gravity values are spatially filtered) collected in the survey is about $1.70 \mathrm{mGal}$ (the standard error is obtained by dividing the root mean square (RMS) difference by $\sqrt{2}$ ).

\section{EGM08 model for Tahiti: optimal degree of spherical} harmonic expansion

In this paper, we choose EGM08 model (Pavlis et al. 2008) to compute the reference values of gravity anomaly and geoidal height in Tahiti for the RCR procedure of geoid modeling. EGM08 is represented by a series of spherical harmonic expansion to degree 2160, with some coefficients exceeding degree 2160. Because of the commission errors in the harmonic coefficients of higher degrees, the optimal use of EGM08 for a best result of geoid modeling in Tahiti is not clear. In this paper, various degrees of expansions, from 720 to 2160, were used to compute gravity anomalies, which were then compared with the new land gravity anomalies to determine the optimal degree of spherical harmonic expansion for the reference values.

Table 4 shows the statistics of differences between land gravity anomalies and those derived from EGM08 to different expansion degrees. There are 387 and 74 point gravity anomalies used for comparison on the islands of Tahiti and Moorea, respectively. The result suggests that the optimal degree of expansion is 1500 , which results in a mean difference of $5.83 \mathrm{mGal}$ and a minimum standard

Table 3 Statistics of gravity differences at crossover points before and after the crossover adjustment (units: mGal)

\begin{tabular}{llllll}
\hline & Max & Min & Mean & RMS & Standard deviation \\
\hline Before & 9.43 & -9.28 & -0.51 & 4.34 & 4.33 \\
After & 4.32 & -5.27 & 0.00 & 2.41 & 2.39 \\
\hline
\end{tabular}

Table 4 Statistics of differences between land gravity anomalies and those from EGM08 expanded to different harmonic degrees (units: mGal)

\begin{tabular}{|c|c|c|c|c|c|c|}
\hline Degree & Area & Max & Min & Mean & RMS & Standard deviation \\
\hline 720 & Tahiti & 89.63 & -31.52 & 6.55 & 22.87 & 21.94 \\
\hline 1080 & & 59.50 & -50.00 & -3.61 & 20.51 & 20.21 \\
\hline 1440 & & 40.62 & -55.49 & -3.24 & 18.59 & 18.33 \\
\hline 1500 & & 40.42 & -53.70 & -3.15 & 18.43 & 18.18 \\
\hline 1800 & & 36.87 & -55.85 & -3.10 & 18.31 & 18.07 \\
\hline 2160 & & 41.38 & -59.18 & -2.88 & 17.70 & 17.48 \\
\hline 720 & Moorea & 146.11 & 75.39 & 101.65 & 102.77 & 15.20 \\
\hline 1080 & & 112.63 & 55.07 & 73.03 & 73.86 & 11.12 \\
\hline 1440 & & 87.59 & 38.17 & 54.90 & 55.59 & 8.80 \\
\hline 1500 & & 85.44 & 39.05 & 53.42 & 54.02 & 8.03 \\
\hline 1800 & & 89.33 & 42.71 & 54.73 & 55.48 & 9.14 \\
\hline 2160 & & 99.25 & 36.61 & 57.46 & 59.12 & 14.01 \\
\hline 720 & All & 146.11 & -31.52 & 21.64 & 46.00 & 40.64 \\
\hline 1080 & & 112.63 & -50.00 & 8.56 & 34.92 & 33.89 \\
\hline 1440 & & 87.59 & -55.49 & 5.98 & 27.95 & 27.33 \\
\hline 1500 & & 85.44 & -53.70 & 5.83 & 27.37 & 26.77 \\
\hline 1800 & & 89.33 & -55.85 & 6.08 & 27.76 & 27.12 \\
\hline 2160 & & 99.25 & -59.18 & 6.70 & 28.60 & 27.84 \\
\hline
\end{tabular}

deviation of $26.77 \mathrm{mGal}$. Gravity anomalies from harmonic expansions of EGM08 to degrees 1500-2160 result in biases of about $50-60 \mathrm{mGal}$ in the island of Moorea. These large biases are the result of lacking ground gravity data around Tahiti when constructing EGM08 and will affect the interpretation of the Moorea volcanism; see also the discussions for Table 7 below.

\section{New digital topography model of Tahiti for RTM gravity and geoid effects}

A high-precision and high-resolution DTM helps to pick up the high-frequency component of a geoid model through the use of residual terrain model (RTM; Forsberg 1984). We constructed a new Tahiti DTM on a $1 " \times 1$ " grid by combining $5 \times 5 \mathrm{~m}$ land elevations from Service de l'Aménagement et de l'Urbanisme of French Polynesia and $30 " \times 30^{\prime \prime}$ ocean depths from the SRTM30_PLUS product (http://topex.ucsd.edu/marine_topo/mar_topo.html;

Becker et al. 2009; Sandwell et al. 2014). Figure 4 shows the new DTM, which reveals detailed topographic features of Tahiti. The new DTM was assessed by the elevations at 54 benchmarks observed by precision leveling. Table 5 summarizes the statistics of the differences between the observed and DTM-interpolated elevations. This assessment shows that the DTM is at the 1-m level accuracy. The new DTM shows that Tahiti is about $6500 \mathrm{~m}$ high, 


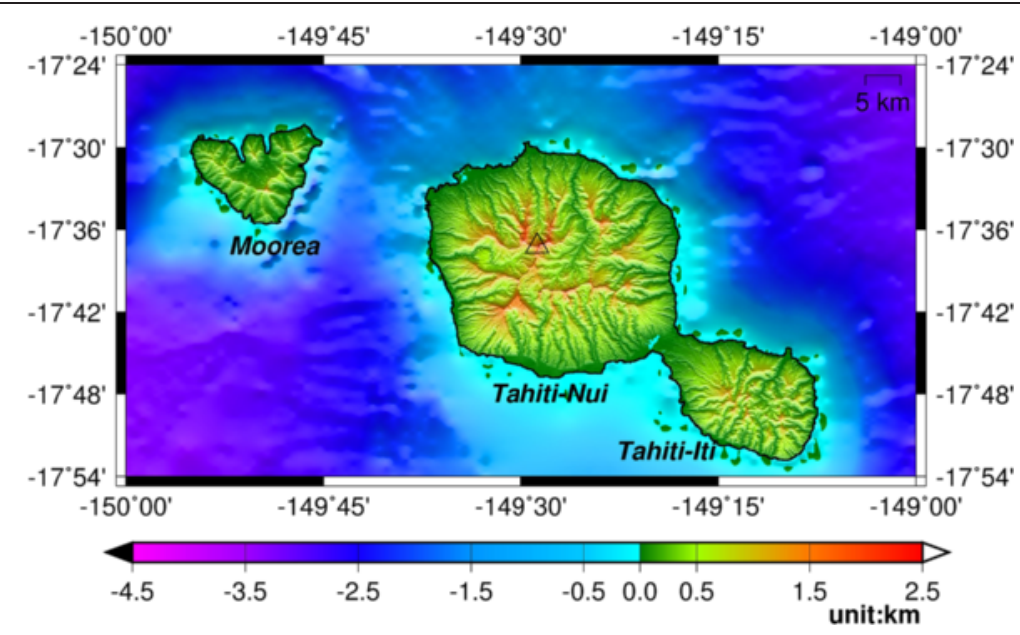

Fig. 4 The new DTM of Tahiti. The island of Tahiti is divided into two parts: the northwestern part "Tahiti-Nui" and the southeastern part "Tahiti-lti". The highest Mt. Orohena in Tahiti is $2241 \mathrm{~m}$. The triangle represents Mt. Orohena

counting from the mean seafloor depth of $4300 \mathrm{~m}$ (Clouard et al. 2000) to its highest peak, Mt. Orohena, whose elevation is $2241 \mathrm{~m}$.

To compute the RTM-derived gravity and geoid effects, the $1 " \times 1$ " grid was re-sampled onto a 9" $\times 9$ " grid. It turns out a 9" $\times 9$ " grid is sufficiently good for our geoid modeling and is regarded as the "true terrain" in all computations below. A smoothed terrain was obtained by applying the Gaussian filter to the 1 " $\times 1$ " grid using an 18-km window size. A RTM was obtained by subtracting the smoothed topography from the true terrain. The technique used for computing the RTMderived gravity and geoid effects is detailed in Hwang et al. (2003). In order to assess the accuracy of the geoid models in this paper, we collected GPS and leveling data at 48 benchmarks on the island of Tahiti and at 6 benchmarks on Moorea. The orthometric heights were based on the vertical datum of Nivellement General de Polynesie Francaise (NGPF; Leveling General of French Polynesia; Service Hydrographique et Océanographique de la Marine 2012), which uses the mean sea level at Matavai bay as the origin of height for the island of Tahiti, and the mean sea level at Papetoai dock for the island of Moorea. We compute the geometric geoidal heights by

Table 5 Statistics of differences between observed heights and those from the 1" $\times 1$ " DTM at 54 leveling benchmarks (units: m)

\begin{tabular}{lllll}
\hline Max & Min & Mean & RMS & Std. dev. \\
\hline 4.428 & -4.752 & 0.001 & 0.287 & 0.287 \\
\hline
\end{tabular}

$$
N_{\text {geo }}=h-H_{o}
$$

where $h$ and $H_{o}$ are the ellipsoidal heights and the orthometric heights, respectively. Here, the "geometric" geoidal heights are computed from geometric measurements. In contrast, the "gravimetric" geoidal heights are purely based on gravity measurements. The orthometric heights were collected along the leveling routes in our levelinggravity surveying campaign. Orthometric corrections (Heiskanen and Moritz 1967) have been applied to the raw differential heights, so the orthometric heights used in this paper are regarded as "true" orthometric heights (subject to an offset between the vertical datum of Tahiti and the geoid). The ellipsoidal heights of the 48 benchmarks were determined by the Leica Geo Office software using GPS static observations on the benchmarks. The session length for GPS measurements is $24 \mathrm{~h}$, resulting in a 1-cm accuracy in the ellipsoidal heights. The geodetic reference system (GRS) of Tahiti is the Réseau Géodésique de Polynésie Française (RGPF; Geodetic Network French Polynesia; Service Hydrographique et Océanographique de la Marine 1999). The RGPF is a local solution of the ITRF 92 geodetic system and then is also based onto the GRS80 ellipsoid. In conclusion, apart from a datuminduced bias in the orthometric heights, the geometric geoidal heights have a $1-\mathrm{cm}$ accuracy.

\section{Downward continuation of airborne gravity by band- limited least-squares collocation}

For combination with different gravity datasets and for geoid modeling, the Tahiti airborne gravity data was downward continued to sea level. Downward continuation is an unstable process and will amplify short-wavelength noises in the data, requiring a low-pass filter to smooth the 
resulting gravity. In this paper, we used the procedure described in Hwang et al. (2006) for the downward continuation and filtering. The airborne gravity contains gravity signals only over certain wave bands, depending on the filter used, i.e., band-limited. The band-limited gravity anomaly $\Delta g_{H}^{\bar{l}+b}$ at a flight altitude $H$ can be expressed as

$$
\Delta g_{H}^{\bar{l}+b}(r, \psi, \lambda)=\frac{G M}{R^{2}} \sum_{n=2}^{\bar{l}+b}(n-1)\left(\frac{R_{E}}{r}\right)^{n+2} T_{n}(\psi, \lambda)
$$

where $(\psi, \lambda)$ are geocentric latitude and longitude, $G M$ denotes the product of the Newtonian constant and the earth's mass, $T_{n}$ is the surface spherical harmonic of degree $n, \bar{l}+b$ is the resolvable degree corresponding to the resolvable wavelength of the airborne gravity, $\bar{l}$ is the degree of expansion of the reference GGM (the optimal case, $\bar{l}$ $=1500$, in Table 4), $R_{E}$ is the mean radius of the earth and $r=R_{E}+H$ is the radius of a sphere at a flight altitude $H$.

The band-limited gravity anomaly contains gravity signal components at wavelengths from degree 2 to $\bar{l}+b$. The relation between $\bar{l}+b$ and the resolvable wavelength $\lambda_{H}$ of the airborne gravity data is (Seeber 2003):

$$
\bar{l}+b=\frac{2 \pi\left(R_{E}+H\right)}{\lambda_{H}}
$$

Because of the limited frequency contents of the Tahiti airborne gravity, we used BL-LSC (Moritz 1978) to carry out the downward continuation and the combination with the altimeter-derived gravity. Hwang et al. (2014) has used BL-LSC to combine gravity data of different spatial resolutions and accuracies in Taiwan, which, like Tahiti, is an island surrounded by seas. In Hwang et al. (2014), the downward continuation (for the airborne gravity dataset) and merging of all gravity datasets were carried out in a one-step procedure. In order to assess the gravity contribution from each of the datasets in this paper, we used a sequential combination procedure described below, rather than the one-step procedure. The BL-LSC computes a residual gravity anomaly $\delta \Delta g_{Z}^{\bar{l}+b}$ at a different altitude $Z$ (other than the original altitude $H$ ) by

$$
\delta \Delta g_{Z}^{\bar{l}+b}=\mathbf{C}_{\Delta g_{Z} \Delta g_{H}}^{\bar{l}+b}\left[\mathbf{C}_{\Delta g_{H} \Delta g_{H}}^{\bar{l}+b}+\mathbf{D}_{\Delta g_{H}}\right]^{-1} \boldsymbol{\delta} \Delta \mathbf{g}_{H}^{\bar{l}+b}
$$

where $\boldsymbol{\delta} \Delta \mathbf{g}_{H}^{\bar{l}+b}$ is a vector containing residual gravity anomalies at the original altitude $H, \mathbf{C}_{\Delta g_{H} \Delta g_{H}}^{l+b}$ is the autocovariance matrix of the airborne gravity anomalies, $\mathbf{C}_{\Delta g_{Z} \Delta g_{H}}^{l+b}$ is the cross-covariance matrix (in this case, a row vector) between the airborne gravity anomaly and the predicted gravity anomaly, and $\mathbf{D}_{\Delta g_{H}}$ is the diagonal error covariance matrix of the airborne gravity anomalies at the altitude $H$.
The residual airborne gravity anomaly $\delta \Delta g_{H}^{\bar{l}+b}$ at the altitude $H$ can be computed by

$$
\delta \Delta g_{H}^{\bar{l}+b}=\Delta g_{H}^{\bar{l}+b}-\Delta g_{\mathrm{GGM}}^{\bar{l}}-F\left(\Delta g_{\mathrm{RTM}}\right)
$$

where $\Delta g_{\mathrm{GGM} \bar{l}}$ is the contribution of a GGM to degree $l$ (1500, in Table 4), $\Delta g_{\text {RTM }}$ is the RTM-derived gravity and $F$ is the same low-pass filtering as the one used in the reduction of airborne gravity observations. $\Delta g_{\mathrm{GGM} \bar{l}}$ and $\Delta g_{\mathrm{RTM}}$ are computed at the flight altitude $H$. The final gravity anomaly $\Delta g_{Z}^{\bar{l}+b}$ at the altitude $Z$ is the summation of the downward-continued residual gravity anomaly $\delta \Delta g_{Z}^{\bar{l}+b}$ in Eq. (4), the GGM-derived gravity and the RTM-derived gravity. The latter two were computed at the altitude $Z$.

We modeled the needed covariance functions by GRAVSOFT (Forsberg and Tscherning 2008), which were then used to compute the elements of all matrices in Eq. (4). The band-limited covariance functions between two gravity anomalies, between gravity anomaly and geoidal height, and between two geoidal heights for points $P$ and $Q$ can be respectively expressed as (Tscherning and Rapp 1974)

$$
\begin{aligned}
& C_{\Delta g \Delta g}^{\bar{l}+b}(P, Q)= \\
& \sum_{n=2}^{\bar{l}} \delta C_{n} s^{n+2} P_{n}\left(\cos \Psi_{P Q}\right)+\sum_{n=\bar{l}+1}^{\bar{l}+b} C_{n} s^{n+2} P_{n}\left(\cos \Psi_{P Q}\right)
\end{aligned}
$$

$$
\begin{aligned}
& C_{\Delta g N}^{\bar{l}+b}(P, Q)= \\
& \frac{R_{B}^{2}}{r_{P} \gamma_{Q}}\left[\sum_{n=2}^{l} \frac{\delta C_{n}}{n-1} s^{n+1} P_{n}\left(\cos \Psi_{P Q}\right)+\sum_{n=\bar{l}+1}^{\bar{l}+b} \frac{C_{n}}{n-1} s^{n+2} P_{n}\left(\cos \Psi_{P Q}\right)\right]
\end{aligned}
$$

$$
\begin{aligned}
& C_{N N}^{\bar{l}+b}(P, Q)= \\
& \frac{R_{B}^{2}}{\gamma_{P} \gamma_{Q}}\left[\sum_{n=2}^{\bar{l}} \frac{\delta C_{n}}{(n-1)^{2}} s^{n+1} P_{n}\left(\cos \Psi_{P Q}\right)+\sum_{n=\bar{l}+1}^{\bar{l}+b} \frac{C_{n}}{(n-1)^{2}} s^{n+1} P_{n}\left(\cos \Psi_{P Q}\right)\right]
\end{aligned}
$$

and

$$
s=\frac{R_{B}^{2}}{r_{p} r_{Q}}=\frac{\left(R_{E} S\right)^{2}}{\left(R_{E}+H_{p}\right)\left(R_{E}+H_{Q}\right)}
$$

where $\delta C_{n}$ is the error degree variance at $n, C_{n}$ is the signal degree variance, $r_{P}$ and $r_{\mathrm{Q}}$ are the geocentric distances for $P$ and $Q, \gamma_{P}$ and $\gamma_{Q}$ are the normal gravity values, $R_{B}$ is the radius of the Bjerhamar's sphere, $S=R_{B} / R, \Psi_{P Q}$ is the spherical distance between $P$ and $Q$, and $P_{n}$ is the associated Legendre function (Heiskanen and Moritz 1967). The error degree variance is computed by (Hwang 1989) 


$$
\delta C_{n}=\left(\frac{G M}{R_{E}^{2}}\right)^{2}(n-1)^{2}\left(\frac{R_{E}}{R_{B}}\right)^{2(n+2)} \sum_{m=0}^{n}\left(\bar{\varepsilon}_{c n m}^{2}+\bar{\varepsilon}_{\text {snm }}^{2}\right)
$$

where $\bar{\varepsilon}_{\mathrm{cnm}}$ and $\bar{\varepsilon}_{\mathrm{snm}}$ are the error variances for cosine and sine terms of the corresponding EGM08 geopotential coefficients, respectively. The signal degree variance is based on Model 4 of Tscherning and Rapp (1974):

$$
C_{n}=\frac{A(n-1)}{(n-2)(n+B)}, n>2
$$

By definition, $C_{2}$ is zero. We used the modules EMPCOV and COVIT in GRAVSOFT to adjust the parameters $A$ and $B$ in Eq. (11) and $S$ in Eq. (9). For the Tahiti airborne gravity survey, we adopted the following values: $3.223 \mathrm{~km}$ for flight height $(H), 6371 \mathrm{~km}$ for mean Earth radius $(R)$, and $11 \mathrm{~km}$ for the resolvable wavelength $\left(\lambda_{H}\right)$.Using Eq. (3), we obtain $\bar{l}+b \approx 3600$, which corresponds to a spatial resolution of $5.5 \mathrm{~km}$ (half-wavelength). The long-wavelength contribution is from the EGM08 to degree $\bar{l}=1500$ (Table 4). Figure 5 shows the covariance functions between two gravity anomalies based on the original parameters of the Tscherning and Rapp Model $4\left(A=425.28 \mathrm{mGal}^{2}, B=24, S=0.999617\right)$ and the adjusted parameters $\left(A=388.55 \mathrm{mGal}^{2}, B=24\right.$, $S=0.999619)$ for Tahiti. At spherical distances lower than $0.025^{\circ}$, the differences between the summation to $\bar{l}+b=\infty$ and $\bar{l}+b=3600$ are larger, compared to the differences at spherical distances greater than $0.025^{\circ}$. This indicates the near field data will have more influence on the result than the far field data when the covariance function is changed. The use of the adjusted Model 4 leads to smaller variances in the covariance functions in Eqs. (6), (7), and (8). The correlation lengths remain at about $0.05^{\circ}$ for both the cases of the original and the adjusted Model 4 signal degree variances.

\section{Constructing new free-air and Bouguer gravity anomaly grids and geoid grids}

The free-air gravity anomaly and gravimetric geoid grids of Tahiti were computed by the BL-LSC in a classic RCR procedure, which splits a total signal into three spectral components as:

$$
\begin{aligned}
& \Delta g=\Delta g_{\mathrm{GGM}}^{1500}+\Delta g_{\text {medium }}^{3600}+\Delta g_{\text {short }}^{\infty} \\
& N_{\text {grav }}=N_{\mathrm{GGM}}^{1500}+N_{\text {medium }}^{3600}+N_{\text {short }}^{\infty}+N_{\text {quasi }}
\end{aligned}
$$

where $\Delta g_{\mathrm{GGM}}^{1500}$ and $N_{\mathrm{GGM}}^{1500}$ are gravity anomaly and geoidal height from EGM08 to degree 1500, $\Delta g_{\text {Medium }}^{3600}$ and $N_{\text {Medium }}^{3600}$ are the medium-wavelength components, corresponding to the contributions from degrees 1501-3600, $\Delta g_{\text {short }}^{\infty}$ and $N_{\text {short }}^{\infty}$ are the short-wavelength components, representing the contributions beyond degree 3600 . The last term $N_{\text {quasi }}$ in Eq. (13) is to account for the difference between the quasi-geoid and the geoid, the latter being used to define an orthometric height system. $N_{\text {quasi }}$ is computed as (Hsiao 2007)

$$
N_{\text {quasi }}=-\frac{2 \pi G \rho}{\gamma} H_{o}^{2}
$$

where $\rho=2.80 \mathrm{~g} / \mathrm{cm}^{3}$ is the mean volcanic load density for Tahiti and Moorea (Clouard et al. 2000), $\gamma$ is the normal gravity of the GRS80 ellipsoid (Eq. (7)) and $H_{o}$ is the orthometric height (practically, it is the height interpolated from the new DTM). $N_{\text {quasi }}$ at sea was set to zero.

By the BL-LSC, we computed the medium and short wavelength gravity and geoid components as follows. The medium-wavelength components of gravity and geoid were computed as
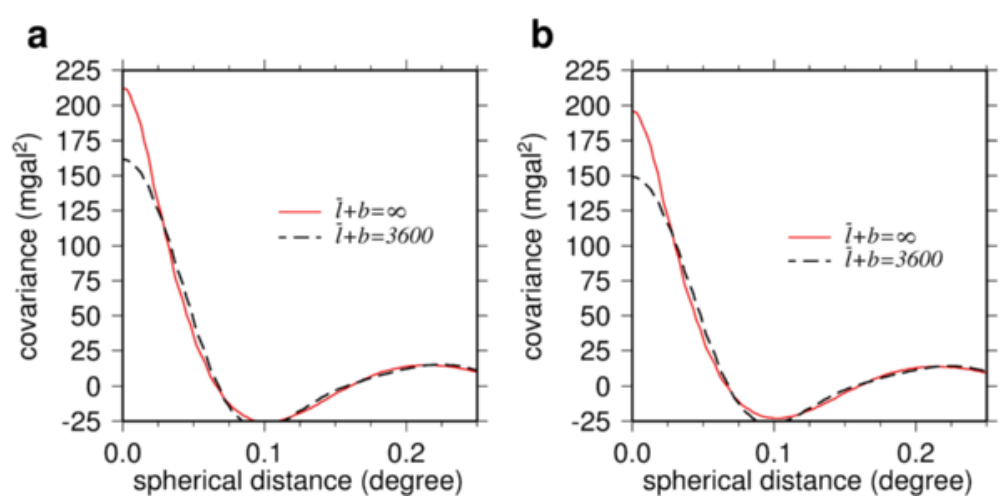

Fig. 5 Covariance functions for gravity anomaly. a The original Tscherning and Rapp covariance model $\mathbf{b}$ The adjusted model, up to $\bar{l}+b=\infty$ and 3600 


$$
\Delta g_{\text {medium }}^{3600}=\mathbf{C}_{\Delta g \Delta g}^{3600}\left[\begin{array}{cc}
\mathbf{C}_{\Delta g \Delta g}^{3600}+\mathbf{D}_{\Delta g_{\text {down-air }}} & 0 \\
0 & \mathbf{C}_{\Delta g \Delta g}^{3600}+\mathbf{D}_{\Delta g_{\text {occan }}}
\end{array}\right]^{-1}\left[\begin{array}{c}
\boldsymbol{\delta} \Delta \mathbf{g}_{\text {down-air }} \\
\mathbf{\delta} \Delta \mathbf{g}_{\text {ocean }}
\end{array}\right]
$$

$$
N_{\text {medium }}^{3600}=\mathbf{C}_{\Delta g \Delta g}^{3600}\left[\begin{array}{cc}
\mathbf{C}_{\Delta g \Delta g}^{3600}+\mathbf{D}_{\Delta g_{\text {down-air }}} & 0 \\
0 & \mathbf{C}_{\Delta g \Delta g}^{3600}+\mathbf{D}_{\Delta g_{\text {ocean }}}
\end{array}\right]^{-1}\left[\begin{array}{c}
\mathbf{\delta} \Delta \mathbf{g}_{\text {down-air }} \\
\mathbf{\delta} \Delta \mathbf{g}_{\text {ocean }}
\end{array}\right]
$$

where vectors $\boldsymbol{\delta} \Delta \mathbf{g}_{\text {down-air }}$ and $\boldsymbol{\delta} \Delta \mathbf{g}_{\text {ocean }}$ contain the residual downward-continued airborne gravity anomalies and the residual marine gravity anomalies, the latter being obtained by subtracting the degree-1500 EGM08 gravity from the altimeter-derived gravity anomalies.
The short-wavelength components were computed from the residual land gravity anomaly and the RTM-derived gravity anomaly as

$$
\begin{gathered}
\Delta g_{\text {short }}^{\infty}=\mathbf{C}_{\Delta g \Delta g}^{\infty}\left[\begin{array}{cc}
\mathbf{C}_{\Delta g \Delta g}^{\infty}+\mathbf{D}_{\Delta g_{\text {land }}} & 0 \\
0 & \mathbf{C}_{\Delta g \Delta g}^{\infty}+\mathbf{D}_{\Delta g_{\mathrm{RTM}}}
\end{array}\right]^{-1}\left[\begin{array}{c}
\boldsymbol{\delta} \Delta \mathbf{g}_{\text {land }} \\
\Delta \mathbf{g}_{\mathrm{RTM}}
\end{array}\right] \\
(17) \\
N_{\text {short }}^{\infty}=\mathbf{C}_{N \Delta g}^{\infty}\left[\begin{array}{cc}
\mathbf{C}_{\Delta g \Delta g}^{\infty}+\mathbf{D}_{\Delta g_{\text {land }}} & 0 \\
0 & \mathbf{C}_{\Delta g \Delta g}^{\infty}+\mathbf{D}_{\Delta g_{\mathrm{RTM}}}
\end{array}\right]^{-1}\left[\begin{array}{c}
\boldsymbol{\delta} \Delta \mathbf{g}_{\text {land }} \\
\Delta \mathbf{g}_{\mathrm{RTM}}
\end{array}\right]
\end{gathered}
$$

where vectors $\boldsymbol{\delta} \Delta \mathbf{g}_{\text {land }}$ and $\Delta \mathbf{g}_{\mathrm{RTM}}$ are the residual land and RTM-derived gravity anomalies, the former being

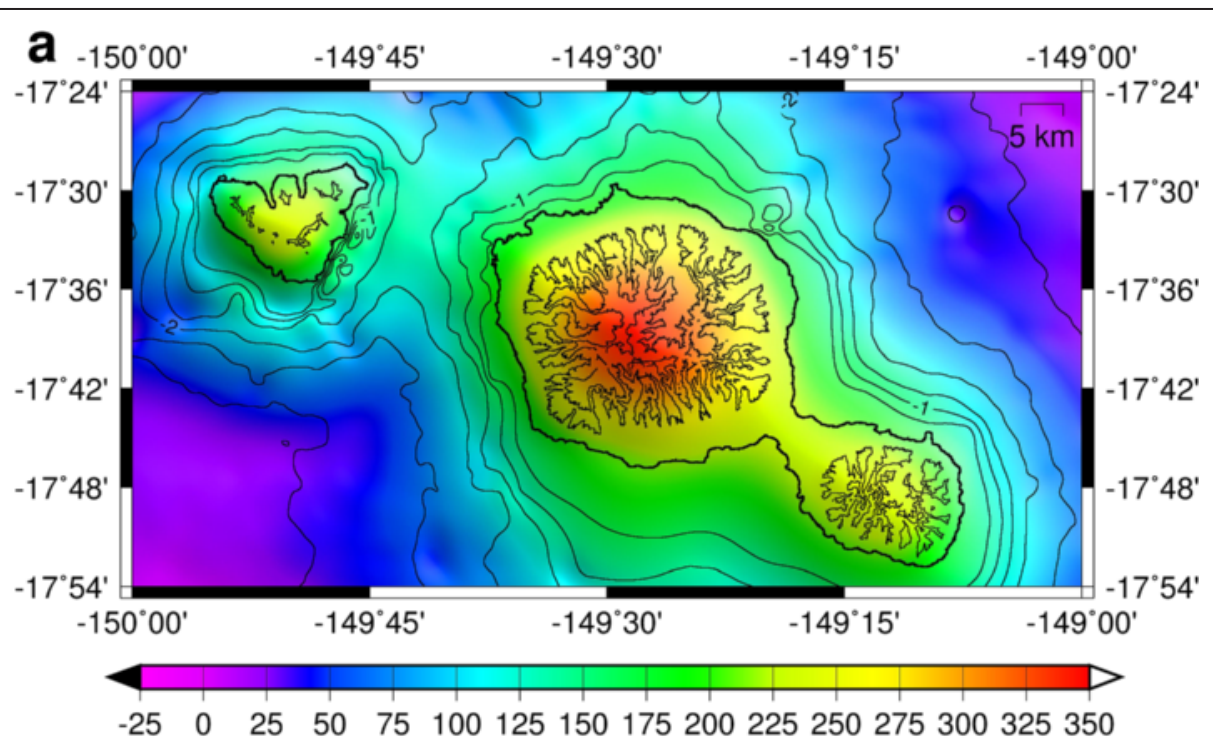

b

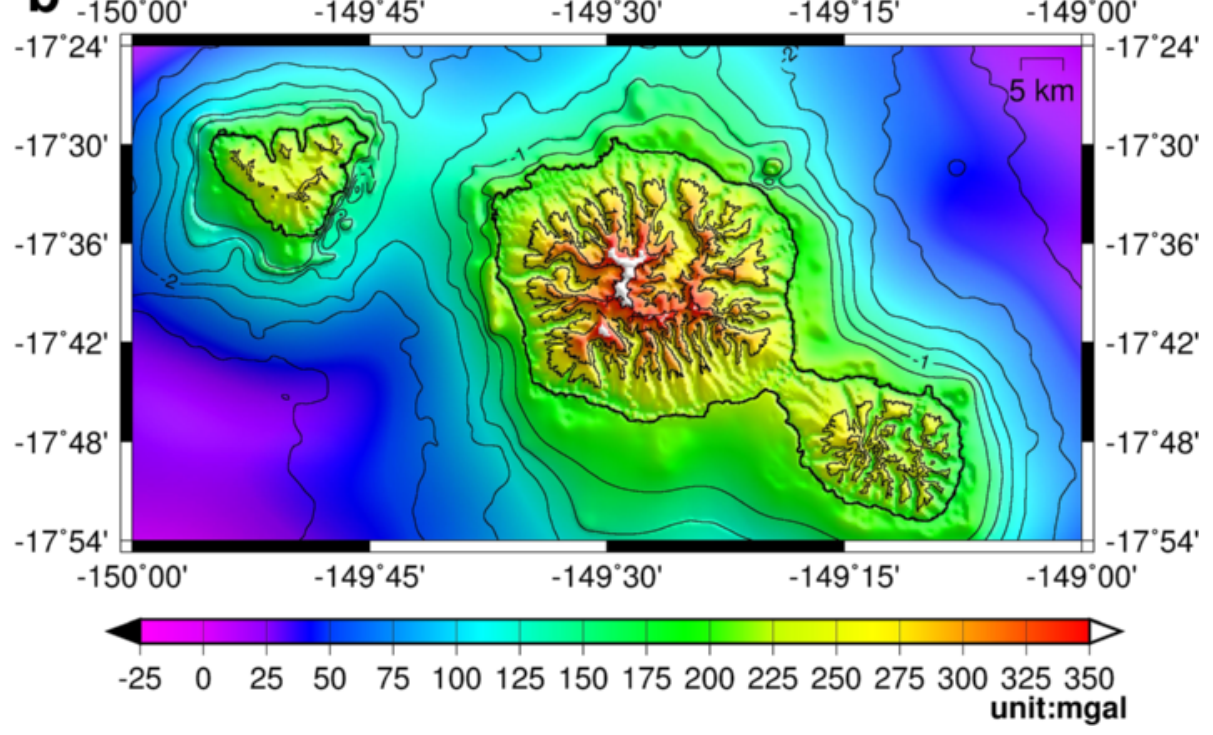

Fig. 6 Free-air gravity anomaly models of Tahiti. The two models (see Table 7) are from: a EGM08 to degree 1500, airborne and altimeter gravity $(1500+a+0)$. b All datasets $(1500+a+o+t+l)$. The topographic contours are shown at a 500-m interval 


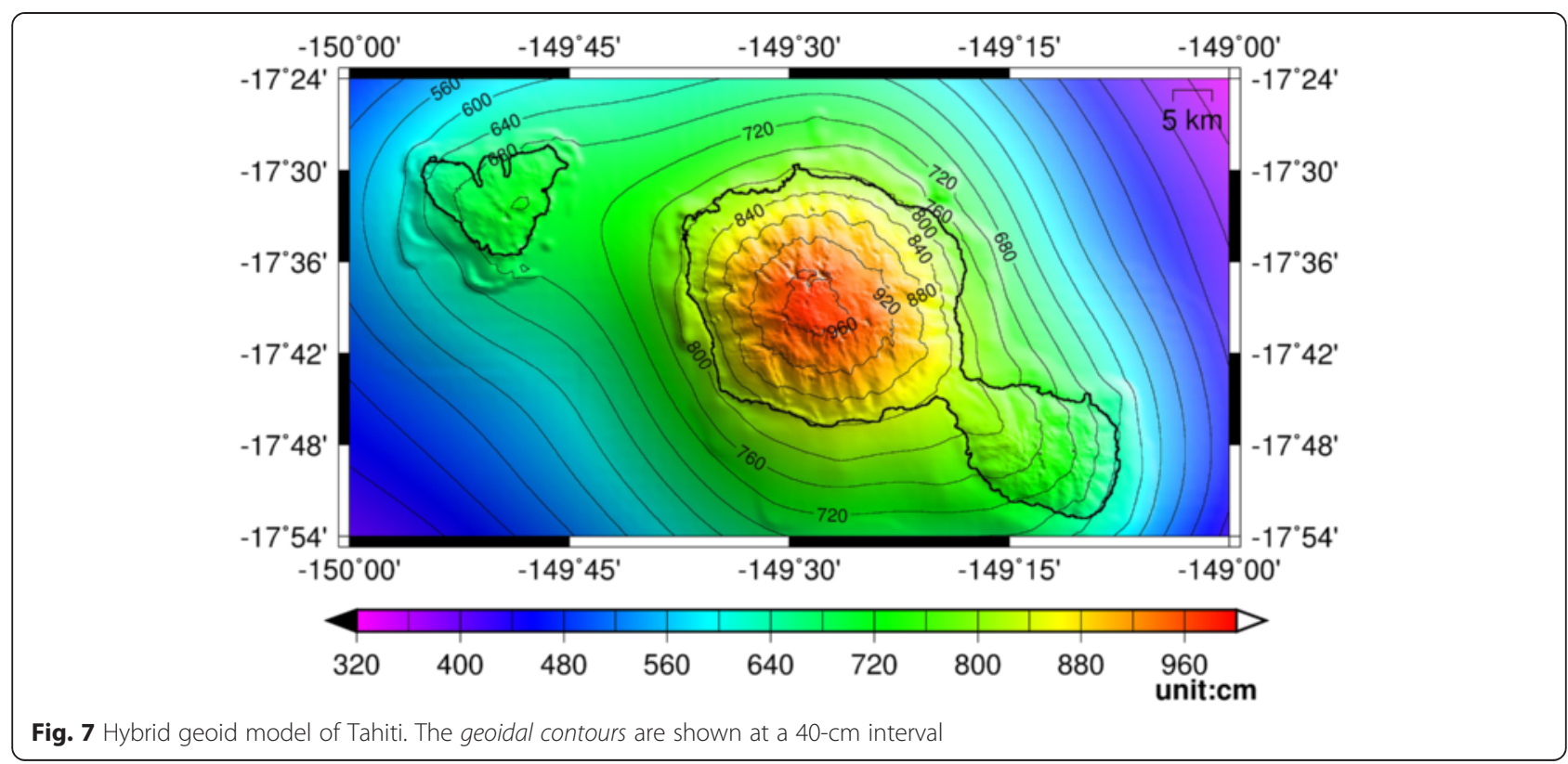

obtained by subtracting the medium wavelength gravity component (Eq. (15)) and long-wavelength gravity components (due to EGM08 to degree 1500) from the land gravity anomalies.

The hybrid geoid model was constructed by combining the gravimetric and the geometric geoidal height using the following step. First, we computed the following quantities

$$
\begin{aligned}
& \delta N_{\text {grav }}=N_{\text {grav }}-N_{\mathrm{GGM}}^{1500}-N_{\text {quasi }} \\
& \delta N_{\text {geo }}=N_{\text {geo }}-N_{\mathrm{GGM}}^{1500}-N_{\text {quasi }}
\end{aligned}
$$

Then, the residual hybrid geoidal height was computed as

$$
\delta N_{\text {hybrid }}=\mathbf{C}_{N N}\left[\begin{array}{cc}
\mathbf{C}_{N N}+\mathbf{D}_{N_{\text {grav }}} & 0 \\
0 & \mathbf{C}_{N N}+\mathbf{D}_{N_{\text {geo }}}
\end{array}\right]^{-1}\left[\begin{array}{l}
\boldsymbol{\delta} \mathbf{N}_{\text {grav }} \\
\mathbf{\delta}_{\text {geo }}
\end{array}\right]
$$

The final hybrid geoidal height was computed as

$$
N_{\text {hybrid }}=N_{\mathrm{GGM}}^{1500}+\delta N_{\text {hybrid }}+N_{\text {quasi }}
$$

We also computed a Bouguer anomaly (BA) grid of Tahiti in both the land and oceanic regions by the method of Gaussian quadrature (Hwang et al. 2003, 2014). Using the 9" $\times 9$ " DTM of Tahiti and the mean volcanic load density mentioned in Eq. (14), we first evaluated the gravity effects due to the land mass above the mean sea level and the oceanic mass due to the

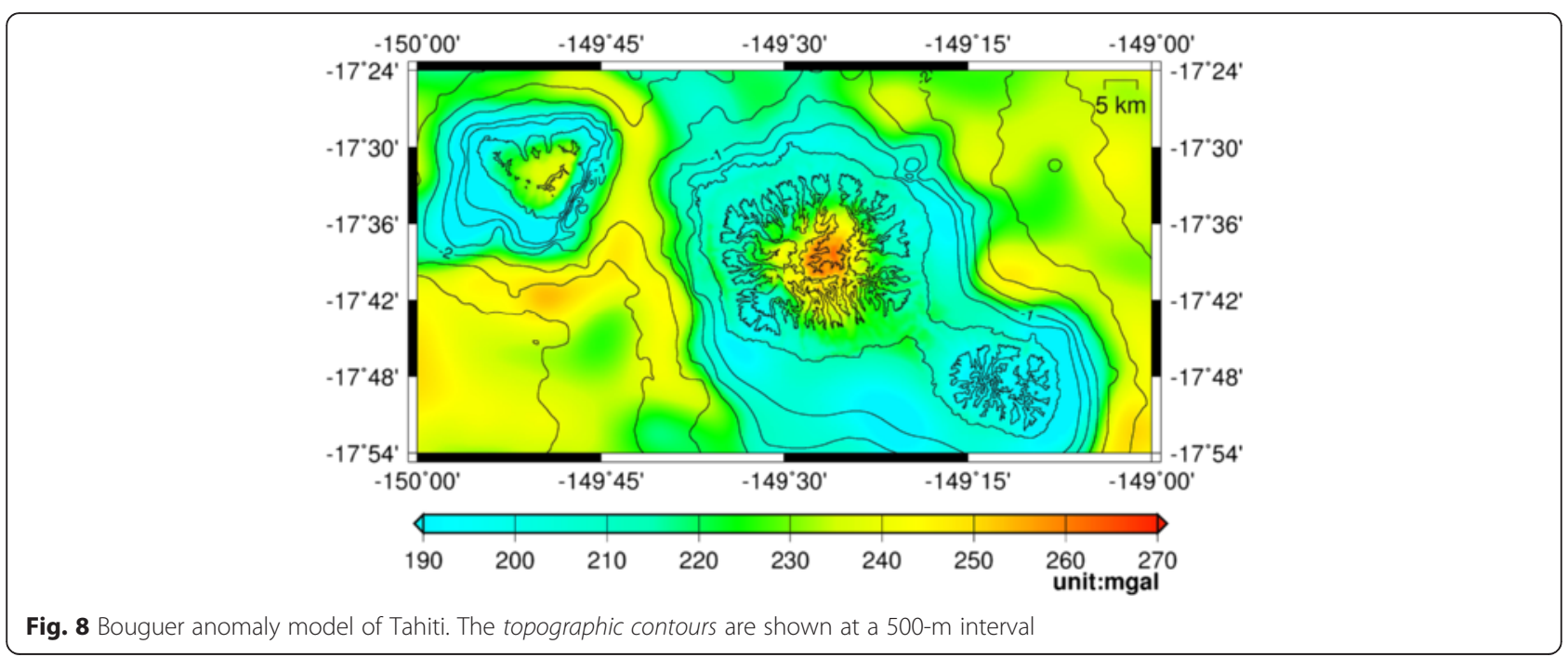


density differences between the mean rock and the sea water. Such gravity effects were then removed from the free-air gravity anomalies to obtain the BAs.

\section{Results and discussion}

In this paper, we constructed four new gravity-related models for Tahiti: free-air gravity anomaly, Bouguer anomaly, gravimetric geoid, and hybrid geoid. They are all given on a 9" $\times 9$ " grid. Figure 6 a shows the free-air gravity anomaly model from the EGM model (to degree 1500), airborne and altimeter gravity, and Fig. 6b shows the model from all datasets (airborne, altimeter, terrain, and land data). The difference between Fig. 6a and 6b highlights the contributions of RTM-derived and land gravity data, which contribute the high-frequency components of the gravity field. Figure 7 shows the hybrid geoid model. The gravimetric geoid map is close to the hybrid geoid map, so it is not shown here. Figure 8 shows the BA model. The statistics of the modeled values of free-air gravity anomaly, Bouguer anomaly, and hybrid geoid are summarized in Table 6. After quality assessments, we will present a preliminary discussion on the scientific implications of these models.

First, we assess the quality of the free-air gravity anomaly model. Using ground gravity measurements, we already assessed EGM08 to decide the optimal degree (i.e., 1500) for use in Tahiti (Table 4). Here, we use the same gravity measurements to assess the free-air gravity anomaly models from different combinations of datasets, in comparison to the degree-1500 EGM08 gravity model. Table 7 shows the statistics of the differences between gravity values from ground measurements and those from different combinations of gravity datasets. The RMS difference drops when an additional dataset is incorporated into the previous model that does not use this dataset. For example, the RMS difference is reduced from 27.37 (the degree-1500 EGM08 geoid model) to $18.51 \mathrm{mGal}$ when adding the airborne gravity data and the altimeter-derived gravity data (the case for $1500+a+o$ in Table 7) to the degree-1500 EGM08 gravity model. This shows the benefits from using airborne and altimeter-derived gravity data.

By adding the RTM contribution, the RMS difference is reduced from 18.51 to $8.59 \mathrm{mGal}$ (the case for 1500 $+a+0+t$ in Table 7). This shows the value of the RTM in delivering the high-frequency gravity components.

Table 6 Statistics of new free-air gravity anomalies and hybrid geoidal heights over Tahiti

\begin{tabular}{llllll}
\hline & Max & Min & Mean & RMS & Std. dev. \\
\hline Gravity anomaly (mGal) & 372.39 & -32.08 & 56.26 & 89.09 & 69.08 \\
Bouguer anomaly (mGal) & 269.73 & 145.58 & 231.52 & 232.18 & 17.54 \\
Geoidal height (m) & 9.77 & 1.08 & 4.57 & 4.92 & 1.82 \\
\hline
\end{tabular}

Table 7 Statistics of the differences between land gravity anomalies and modeled values from different combinations of gravity datasets (unit: $\mathrm{mGal}$ )

\begin{tabular}{lllllll}
\hline Model & Area & Max & Min & Mean & RMS & $\begin{array}{l}\text { Standard } \\
\text { deviation }\end{array}$ \\
\hline $1500+$ a+o & Tahiti & 28.97 & -57.76 & -3.91 & 17.35 & 16.92 \\
& Moorea & 51.94 & -15.12 & 16.14 & 23.77 & 17.56 \\
& All & 51.94 & -57.76 & -0.73 & 18.51 & 18.52 \\
$1500+$ a+o+tt & Tahiti & 30.01 & -13.44 & -1.44 & 9.40 & 9.44 \\
& Moorea & 24.72 & -18.03 & 0.53 & 8.41 & 8.42 \\
$1500+$ a+o+t & Tahiti & 4.44 & -2.47 & 0.19 & 0.91 & 0.89 \\
+1 & Moorea & 2.15 & -3.19 & 0.02 & 0.93 & 0.92 \\
& All & 4.44 & -3.19 & 0.16 & 0.91 & 0.90
\end{tabular}

Note: 1500 is the optimal degree of expansion of EGM08 and symbols a, o, t, and I represent gravity datasets from airborne survey, altimeter, RTM, and land survey

Figure 9 shows the RTM-derived gravity values and the residual land gravity values (Eq. (17)) as functions of orthometric height. Most of the gravity differences in Fig. 9 fall between $\pm 10 \mathrm{mGal}$. In general, the absolute difference between the two commonly increases with orthometric height, largely because the roughness of the terrain will increase the uncertainties in the RTM effects. However, along the coastal lines (at low elevations), there are also some large differences (see Fig. 10a below), indicating that the RTM cannot adequately pick up the high-frequency gravity signals in certain parts of the coasts.

The large gravity differences along the coasts can be reduced by adding the new land gravity dataset, which contains gravity measurements in the coastal areas. This reduction is evident by comparing the differences in Fig. 10a (differences between gravity measurements and modeled values from the case of $1500+a+0+t)$ and Fig. 10b (the case from $1500+a+o+t+1)$. Figure 10a shows large gravity differences exceeding $10 \mathrm{mGal}$ (absolute values) along a part of the south coastline of Tahiti-Nui and the northern flank of the isthmus linking Tahiti-Nui and Tahiti-Iti. The incorporation of land gravity values into the gravity model significantly reduces the gravity differences in these two areas (Fig. 10b). In fact, adding the land gravity data in the model results in the least RMS difference of $0.91 \mathrm{mGal}$ (see the case for $1500+\mathrm{a}+\mathrm{o}$ $+t+l$ in Table 7). These assessments suggest that future high-degree geopotential models will benefit from the new airborne and land gravity data collected in this paper for the Tahiti region.

Next, we assess the quality of the gravimetric geoid model (the case for $1500+\mathrm{a}+\mathrm{O}+\mathrm{t}+\mathrm{l}$ in Table 8) and the hybrid geoid model (the case for $1500+a+o+t+l+n$ in Table 8) using 54 geometric geoidal heights. A similar 


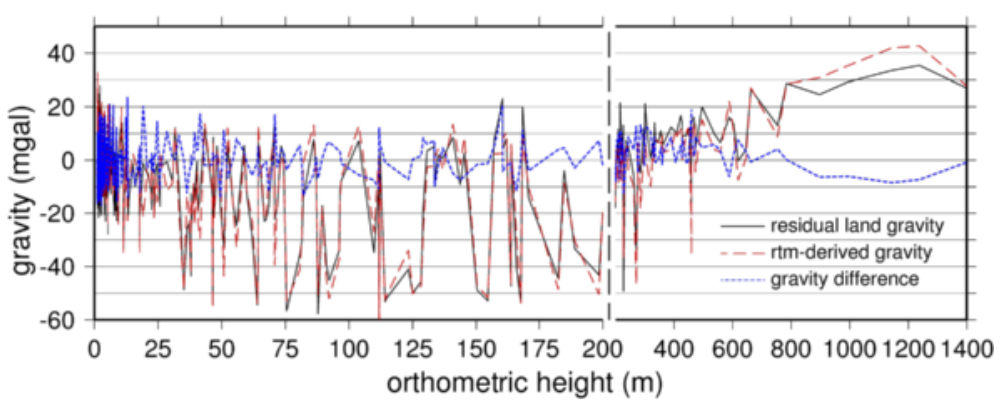

Fig. 9 Residual land gravity anomaly and RTM-derived gravity as functions of orthometric height. The scale along the horizontal axis varies at $x=200 \mathrm{~m}$ to better represent the variations in the data records below $200 \mathrm{~m}$
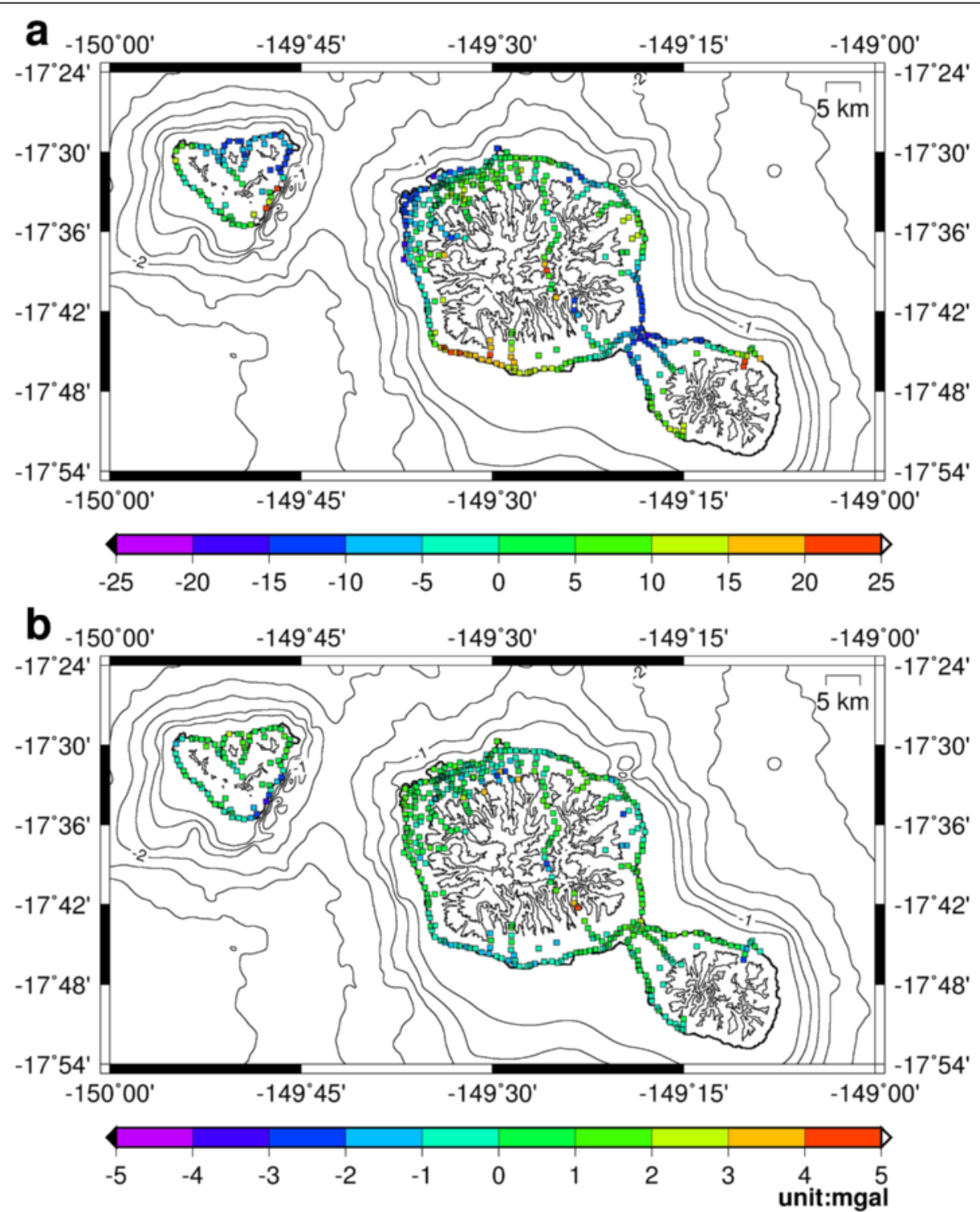

Fig. 10 Differences between land gravity anomalies and modeled values. The modeled values are from two cases (see Table 7). a EGM08 to degree 1500, airborne gravity, altimeter gravity, and RTM $(1500+a+0+t)$. b All datasets $(1500+a+0+t+1)$. The topographic contours are shown at a 500-m interval 
Table 8 Statistics of differences between the modeled geoidal heights and the measured geometric geoidal heights (unit: $\mathrm{cm}$ )

\begin{tabular}{llllll}
\hline Model & Max & Min & Mean & RMS & Standard deviation \\
\hline 1500 & 36.5 & -8.5 & 9.6 & 14.0 & 10.3 \\
$1500+$ a+o & 32.7 & -5.2 & 5.7 & 10.7 & 9.1 \\
$\begin{array}{l}1500+a+0+t \\
1500+a+0+t+1\end{array}$ & 17.2 & -16.9 & 0.7 & 6.4 & 6.4 \\
$\begin{array}{l}\text { (Gravimetric geoid) } \\
\text { 1500+a+o+t+l+n }\end{array}$ & 7.7 & -8.1 & 0.0 & 3.6 & 3.6 \\
(Hybrid geoid) & 1.5 & -1.1 & 0.0 & 0.4 & 0.4
\end{tabular}

Note: for the definitions of symbols a, o, t, and l, see Table $7 ; n$ represents geometric geoidal height evolution of the differences is found for the geoid models that use different gravity datasets. Adding the airborne gravity data and the altimeter-derived gravity data reduces the RMS difference from 14.0 (the degree1500 EGM08 geoid model) to $10.7 \mathrm{~cm}$. The difference is further reduced to $6.4 \mathrm{~cm}$ by adding the RTM-derived gravity data, then to $3.6 \mathrm{~cm}$ by further adding the new land gravity data. Because the geometric geoidal heights at $54 \mathrm{GPS} /$ leveling points are assimilated into the hybrid geoid model, this model yields a RMS difference of $0.4 \mathrm{~cm}$. Figure 11a, b shows the differences between the geometric geoidal heights and those from the gravimetric geoid model and the hybrid geoid model at the 54
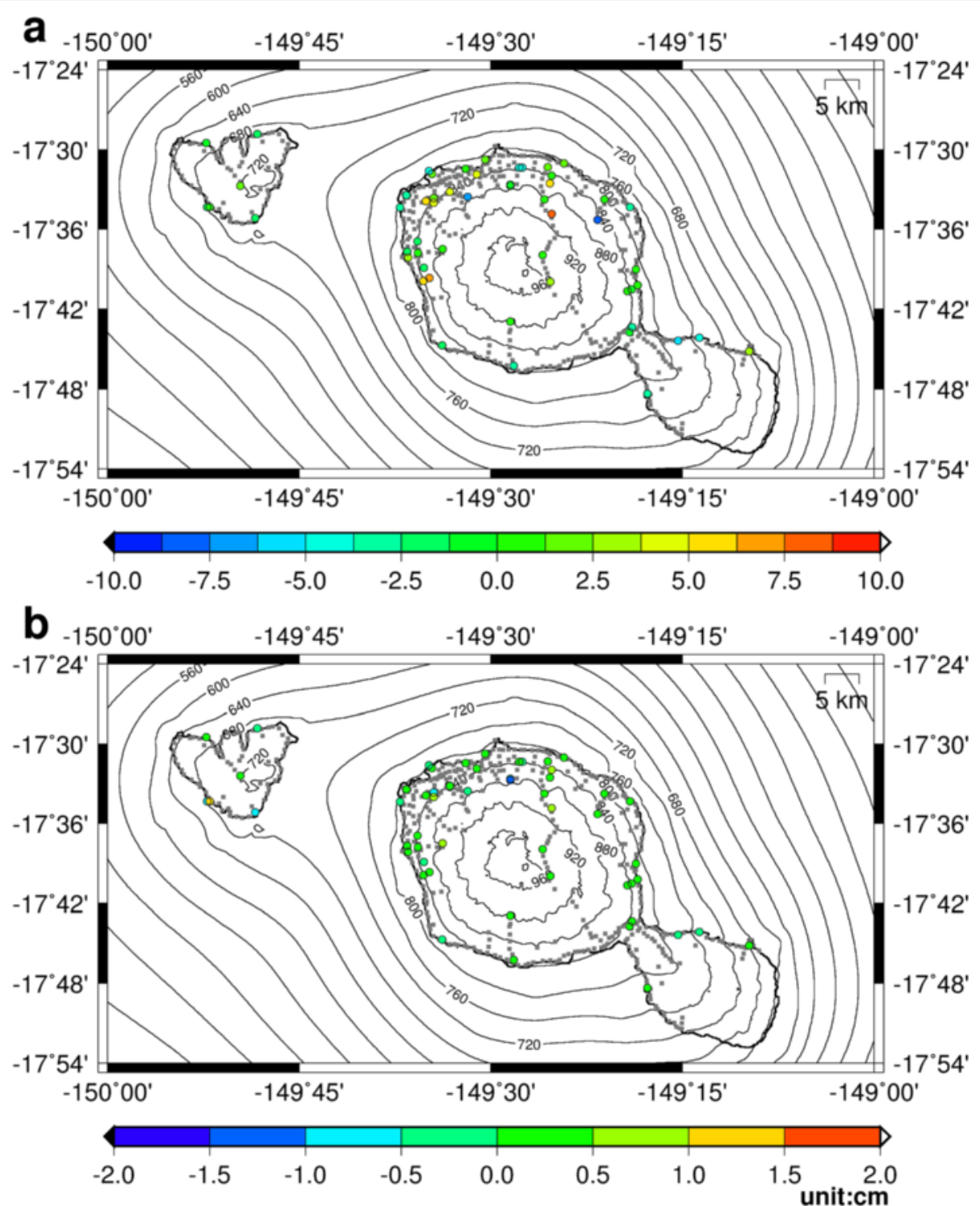

Fig. 11 Differences between geometric geoidal heights and modeled values. The modeled values are from two cases (see Table 8). a The gravimetric geoid model. $\mathbf{b}$ The hybrid geoid model. The geoidal contours are shown at a 40-cm interval. The gray points are the sites of the new land gravity measurements 
points, respectively. Most of the differences in Fig. 11a are within $\pm 3 \mathrm{~cm}$. Because the hybrid geoid model incorporates the observed geometric geoidal heights, naturally it results in smaller geoidal differences as compared to the gravimetric geoid model (Fig. 11a Vs. Fig. 11b).

In comparison to the EGM08 gravity model on land (Fig. 2), the new free-air gravity anomaly model (Fig. 6b) can better reflect the topographic roughness in Tahiti such as the steep, deep valleys that have elevation changes of up to $1000 \mathrm{~m}$. In Tahiti-Nui, the gravity lows (yellow, Fig. 6b) show a wider area of collapse at the northeast sides of the caldera (forming larger valleys, see Fig. 4) than that at the southwest side. The half-round gravity highs constitute the shape of a half-caldera over central Moorea, which is a volcano formed at $1.65 \pm 0.13 \mathrm{Ma}$ ago according to the K-Ar analysis on Moorea rocks (Dymond 1975). The positive geoidal heights (Fig. 7) are caused by the positive mass anomalies from the volcanism.

The new BA structure in Fig. 8 is largely the result of volcano evolution in Tahiti. The remnant magma masses in the centers of Tahiti and Moorea produce large gravity anomalies (over $240 \mathrm{mGal}$ ). In general, the amplitude of gravity decreases toward the seas. The new BA model show a much better definition of spatial patterns of surplus mass associated with the magma reservoirs beneath French Polynesia as highlighted by Clouard et al. (2000). Figure 8 suggests that the BA high on the island of Moorea is deviated from the center of the major caldera by several kilometers. A possible explanation for this deviation is that the mapped caldera is a collapsed feature filled in with lava during a second shield stage as suggested by Flinders et al. (2010), for the positive residual gravity offset of the island of Kaua'i in the Northern Hawaiian Islands. In addition, the maximum BA in the center of Tahiti is larger than that in the center of Moorea, suggesting that the amplitude of BA is in proportion to the size of island. Since the work of Clouard et al. (2000), Clouard et al. (2001), and Hildenbrand et al. (2004, 2006, 2008) have used observations from oceanographic and field campaigns to significantly revise the geomorphological and geological evolution history of the island of Tahiti. These new works suggest that the current state of Tahiti can be oversimplified as the result of two giant north and south flank landsides of the main volcano shield around $0.87 \mathrm{Ma}$, followed by a second shield stage that ended around $0.250 \mathrm{Ma}$. Further investigations using the new BA model of Tahiti may lead to better 3-D modeling of subsurface density structure, but this subject is beyond the scope of this study.

\section{Conclusions}

In this study, we have updated the gravity field of Tahiti by adding datasets from a new airborne mission, a land survey campaign and a new result of altimeter data processing. The new land gravity data collected in 2013 have a mean accuracy of $17 \mu \mathrm{Gal}$ and are on average $17.6 \mathrm{mGal}$ larger than the values acquired in 1994. This 17.6-mGal difference should be investigated to identify potential misinterpretations of the earlier gravity field in Tahiti. The new land and airborne gravity give the gravity highly associated with the volcanism of Moorea, which is not seen in the EGM08 gravity model (compare Figs. 2 and 7). As such, we recommend a new interpretation of the Moorea volcanism using the new gravity model constructed in this paper.

Because the BL-LSC method can best integrate datasets of different accuracies and spatial resolutions, it was used for downward continuing the airborne gravity and for constructing the free-air gravity anomaly and hybrid geoid models of Tahiti. The final free-air gravity anomaly model (last row, Table 7) and the hybrid geoid model (last row, Table 8) match the measured gravity values and geometric geoidal heights to $0.92 \mathrm{mGal}$ and $0.4 \mathrm{~cm}$, respectively. The new BA model of Tahiti can be used for investigations of the subsurface structure of Tahiti.

The most important value of the Tahiti hybrid geoid model is to aid orthometric height determination by sensors such as GPS and Lidar that deliver their heights in the so-called ellipsoidal height system. The use of the geoidal and ellipsoidal heights will yield orthometric heights defined in the same system, providing a consistent height datum for Tahiti. In addition, the new free-air gravity anomaly and BA models can benefit geophysical investigations of Tahiti such as identification of magma chambers, intruding dykes and dense cumulate cores.

\section{Abbreviations}

GGM: global gravity model; BL-LSC: band-limited least-squares collocation; RCR: remove-restore-compute; DTM: digital topography model; SSH: sea surface height; NGDC: National Geophysical Data Center; MOl: Ministry of the Interior; RMS: root mean square; RTM: residual terrain model; BA: Bouguer anomaly.

\section{Competing interests}

The authors declare that they have no competing interests.

\section{Authors' contributions}

JPB and $\mathrm{CH}$ initiated the study, with support from DL (the former chief of Tahiti survey). HCS, MM, CH, PC, and DL collected the data and helped in the data reduction. HCS performed most of the data analysis and drafted the manuscript. MM raised critical issues in the first draft. LS helped in the explanation of the Tahiti Bouguer map. CH finalizes the draft. All authors read and approved the final manuscript.

\section{Acknowledgements}

This study is supported by the Service de l'Aménagement et de I'Urbanisme of French Polynesia "Convention 2684/MAA/AU of June 1st, 2012", MOST/Taiwan, under grant No. 103-2221-E-009-114-MY3 and Ministry of the Interior/Taiwan, under project "Gravity Datum Service (2014)." The comments from two anonymous reviewers greatly improved the quality of this paper. 


\section{Author details}

${ }^{1}$ Research Center for Environmental Changes, Academia Sinica, Taipei 11529, Taiwan. ${ }^{2}$ Department of Civil Engineering, National Chiao Tung University, Hsinchu 300, Taiwan. ${ }^{3}$ Geodesy Observatory of Tahiti, University of French Polynesia, 98702 Faa'a, Tahiti. ${ }^{4}$ Institute of Earth Science, Academia Sinica, Taipei 11529, Taiwan. ${ }^{5}$ Service de l'Aménagement et de I'Urbanisme de la Polynésie française, Papeete, Tahiti.

Received: 6 March 2015 Accepted: 29 July 2015

Published online: 05 August 2015

\section{References}

Becker JJ, Sandwell DT, Smith WHF, Braud J, Binder B, Depner J, Fabre D, Factor J, Ingalls S, Kim S-H, Ladner R, Marks K, Nelson S, Pharaoh A, Sharman G, Trimmer R, VonRosenburg J, Wallace G, Weatherall P (2009) Global bathymetry and elevation data at 30 arc seconds resolution: SRTM30_PLUS. Mar Geod 32(4):355-371

Calmant S, Cazenave A (1987) Anomalous elastic thickness of the ocean lithosphere in the South Central Pacific. Nature 328(6127):236-238

Camacho AG, Montesinos FG, Vieira R (2000) Gravity inversion by means of growing bodies. Geophysics 65(1):95-101. doi:10.1190/1.1444729

Clouard V, Bonneville A, Barsczus HG (2000) Size and depth of ancient magma reservoirs under atolls and islands of French Polynesia using gravity data. J Geophys Res 105:8173-8191. doi:10.1029/1999JB900393

Clouard V, Bonneville A, Gillot PY (2001) A giant landslide on the southern flank of Tahiti Island, French Polynesia. Geophys Res Lett 28(11):2253-2256

Devey CW, Lackschewitz KS, Mertz DF, Bourdon B, Cheminée JL, Dubois J, Guivel C, Hékinian R, Stoffers P (2003) Giving birth to hotspot volcanoes: distribution and composition of young seamounts from the seafloor near Tahiti and Pitcairn islands. Geology 31(5):395-398

Dymond J (1975) K-Ar ages of Tahiti and Moorea, Society Islands, and implications for the hot-spot model. Geology 3:236-240

Flinders AF, Ito G, Garcia MO (2010) Gravity anomalies of the Northern Hawaiian Islands: implications on the shield evolutions of Kaua'i and Niihau. J Geophys Res 115, B08412. doi:10.1029/2009JB006877

Forsberg R (1984) A study of terrain reductions, density anomalies and geophysical inversion methods in gravity field modeling. Tech Rep 355, Department of Geodetic Science and Surveying, Ohio State University, Columbus

Forsberg R, Tscherning CC (2008) An overview manual for the GRAVSOFT geodetic gravity field modelling programs, 2nd edn., Contract Report to JUPEM, Aug 2008

Heiskanen WA, Moritz H (1967) Physical geodesy. W.H. Freeman and Company, San Francisco

Hildenbrand A, Gillot PY, Leroy I (2004) Volcano-tectonic and geochemical evolution of an oceanic intra-plate volcano: Tahiti-Nui (French Polynesia). Earth Planet Sci Lett 217:349-365

Hildenbrand A, Gillot PY, Bonneville A (2006) Offshore evidence for a huge landslide of the northern flank of Tahiti-Nui (French Polynesia). Geochem Geophys Geosyst 7, Q03006. doi:10.1029/2005GC001003

Hildenbrand A, Gillot PY, Marlin C (2008) Geomorphological study of long-term erosion on a tropical volcanic ocean island: Tahiti-Nui (French Polynesia). Geomorphology 93:460-481. doi:10.1016/j.geomorph.2007.03.012

Hsiao YS (2007) Modeling Taiwan geoid using airborne, surface gravity and altimetry data: investigations of downward/upward continuations and Terrain modeling techniques. Dissertation, Department of Civil Engineering, Chiao Tung University, Taiwan

Hwang C (1989) High precision gravity anomaly and sea surface height estimation from Geos-3/Seasat altimeter data. Tech Rep 399, Department of Geodetic Science and Surveying, Ohio State University, Columbus

Hwang C (1998) Inverse Vening Meinesz formula and deflection-geoid formula: applications to the predictions of gravity and geoid over the South China Sea. J Geod 72:304-312

Hwang C, Chang ETY (2014) Seafloor secrets revealed—satellite data reveal formerly unknown tectonic structures. Science 346:32-33. doi:10.1126/science.1260459

Hwang C, Wang CG, Lee LH (2002) Adjustment of relative gravity measurements using weighted and datum-free constraints. Comput Geosci 28:1005-1015

Hwang C, Wang CG, Hsiao YS (2003) Terrain correction computation using Gaussian quadrature: effect of innermost zone. Comput Geosci 29(10):1259-1268
Hwang C, Hsiao YS, Shih HC (2006) Data reduction for scalar airborne gravimetry: theory, computer package and a case study in Taiwan. Comput Geosci 32(10):1573-1584

Hwang C, Hsiao YS, Shih HC, Yang M, Chen KH, Forsberg R, Olesen AV (2007) Geodetic and geophysical results from a Taiwan airborne gravity survey: data reduction and accuracy assessment. J Geophys Res 112 . doi:10.1029/2005JB004220.

Hwang C, Hsu HJ, Chang ETY, Featherstone WE, Tenzer R, Lien TY, Hsiao YS, Shih HC, Jai PH (2014) New free-air and Bouguer gravity fields of Taiwan from multiple platforms and sensors. Tectonophysics 61:83-93. doi:10.1016/j.tecto.2013.11.027

Ihde J (1993) Some remarks on geodetic reference systems in Eastern Europe in preparation of a uniform European geoid. B Geod 67(2):81-85

Leroy I (1994) Evolution des volcans en système de point chaud: Ile de Tahiti, archipel de la Société (Polynésie Française). Dissertation, University Paris XI, Orsay, France

Matsumoto K, Takanezawa T, Ooe M (2000) Ocean tide models developed by assimilating TOPEX/POSEIDON altimeter data into hydrodynamical model: a global model and a regional model around Japan. J Oceanogr 56:567-581

Matsumoto K, Takanezawa T, Ooe M (2001) GOTIC2: a program for computation of oceanic tidal loading effect. J Geod Soc Japan 47:243-248

McNutt M (1998) Superswells. Rev Geophys 36(2):211-244. doi:10.1029/98RG00255

Morelli C, Ganter C, Hankasalo T, McConnell RK, Tanner JB, Szabo B, Uotila U, Whalen CT (1974) The International Gravity Standardization net 1971 International Association of Geodesy, Special publication number, 4, International Association of Geodesy, Paris

Moritz H (1978) Least-squares collocation. Rev Geophys Space Phys 16(3):421-430

Novák P, Heck B (2002) Downward continuation and geoid determination based on band-limited airborne gravity data. J Geod 76(5):269-278

Patriat M, Klingelhoefer F, Aslanian D, Contrucci I, Gutscher MA, Talandier J, Avedik F, Francheteau J, Weigel W (2002) Deep crustal structure of the Tuamotu plateau and Tahiti (French Polynesia) based on seismic refraction data. Geophys Res Lett 29(14):1-1-1-4. doi:10.1029/2001GL013913

Pavlis NK, Holmes SA, Kenyon SC, Factor JK (2008) An Earth Gravitational Model to Degree 2160: EGM2008. General Assembly of the European Geosciences Union, Vienna, Austria, 13-18 April, 2008

Sandwell DT, Müller RD, Smith WHF, Garcia E, Francis R (2014) New global marine gravity model from CryoSat-2 and Jason-1 reveals buried tectonic structure. Science 346:65-67. doi:10.1126/science.1258213

Seeber G (2003) Satellite geodesy, 2nd edn. Walter de Gruyter, Berlin

Service Hydrographique et Océanographique de la Marine (1999) Reseau Geodesique de Polynesie Française. DORIS determination des decalages avec les systemes geodesiques locaux, Synthese des Campagnes (in French)

Service Hydrographique et Océanographique de la Marine (2012) Ouvrage de marée, Références Altimétriques Maritimes, Ports de France métropolitaine et d'outre-mer, Cotes du zéro hydrographique et niveaux caractéristiques de la marée (in French)

Sichoix L, Bonneville A (1996) Prediction of bathymetry in French Polynesia constrained by shipboard data. Geophys Res Lett 23(18):2469-2472. doi:10.1029/96GL02122

Tscherning CC, Rapp RH (1974) Closed covariance expressions for gravity anomalies, geoid undulations, and the deflections of the vertical implied by anomaly degree-variance models. Tech Rep 208, Department of Geodetic Science and Surveying, Ohio State University, Columbus

Yang Y, Hwang C, Hsu HJ, Dongchen E, Wang H (2011) A sub-waveform threshold retracker for ERS-1 altimetry: a case study in the Antarctic Ocean. Comput Geosci 54(1):113-118 